Innovations in Incidence Geometry
Volume 15 (2017), Pages 1-3
ISSN 1781-6475,

\title{
50 Years of Finite Geometry
}

This volume contains some papers submitted on the occasion of Joseph Thas's 70th birthday. A conference on the same occasion took place at Ghent University from 13 to 15 November, 2014.

There were 62 participants and 14 talks. In chronological order, the talks were given by Koen Thas, Francis Buekenhout, Aiden Bruen, Stanley Payne, Bruce Cooperstein, Thomas Connor, Guglielmo Lunardon, Anneleen De Schepper, Bart De Bruyn, Mark Pankov, James Hirschfeld, Jeroen Schillewaert, Antonio Pasini and Hendrik Van Maldeghem.

\section{Legacy}

With Jef Thas turning 70 years, we ended a celebrated period of 50 years of Finite Geometry. Indeed, at the age of 21, Jef, having just obtained his Master's Degree, had already solved some open problems on $k$-arcs in finite projective spaces posed by Beniamino Segre. This was not only the beginning of a very successful career, but also the start of a golden 50 years for Finite Geometry. During that period, Jef was indisputably the leading figure. He solved some of the big open problems with elegant and surprising arguments. Three examples come to mind. The first one is the characterisation of finite classical inversive planes of odd order by one derived affine plane being Desarguesian. The second one is the classification of all flocks of ovoids and hyperbolic quadrics in 3dimensional projective spaces of even order; the difficult odd hyperbolic case was solved by Jef, Laura Bader and Guglielmo Lunardon. The third one is the characterisation of a classical Hermitian curve as a unital in the plane in which the feet of an arbitrary point off the unital are collinear.

Jef is also known for developing the theory of finite generalized quadrangles together with Stan Payne, much beyond the original goal of Jacques Tits, who introduced these structures for the purpose of studying groups of Lie type and simple algebraic groups of relative rank 2 and their mixed variations. Jef proved the most fascinating theorems about them. He discovered the magic relation 
between finite elation generalized quadrangles, projective planes and flocks of cones. This gave incredible boosts to these subjects.

It is impossible to write down all the achievements of Jef Thas. It may be clear that the success of Finite Geometry is to a large extent due to Jef. Conversely, Jef's success is due to the existence of Finite Geometry. For fifty years "Finite Geometry" was a synonym of "Jef Thas." No conference on that subject went by without Jef's presence, and without him announcing a new spectacular result in Finite Geometry. He produced more than 260 papers, of which 100 are as sole author, and several books during that period; they are all substantial! Except for his old mentor Julien Bilo, all his book co-authors lectured at the conference.

Jef's professional career has been more than research. For eleven years, he was chairman of the current Department of Mathematics at Ghent University. He led the department with care and understanding. Before this, he was the chair of his own "Seminar of Finite Geometry and Combinatorics." Remarkably, he became professor at the age of 25 , still due to do his military service at that point! He was a very respected faculty member at Ghent University, and part of almost every committee that had to take some important decisions.

For many of his UGent colleagues, including Hendrik, a professional career would not have existed if Jef had not existed, or if he had not given his unconditional support. (Hendrik: "By being his most frequent co-author, I tried to partially pay back what he gave me, but most of all it proudly shows me that the appreciation goes both ways!")

Jef is a friend to everyone he meets, and has built an incredibly large circle of friends throughout the (fifty) years. This goes beyond age barriers, but is also witnessed through the presence of so many people at the conference, and the willingness of so many people to write a paper in his honour, although the journal is not indexed by the Science Citation Index (yet).

\section{The present volume}

The present volume contains 11 papers, most of them by speakers, on the themes of the conference. Particular topics in Finite Geometry that appear are simple groups, flocks, Grassmannians, polar spaces, unitals, semifields. All these topics are at the heart of Jef's research interests!

The papers are by, in alphabetical order, Francis Buekenhout and Thomas Connor; Bruce Cooperstein; Bart De Bruyn; Bill Cherowitzo, Norm Johnson and Oscar Vega; Alice Hui; Dirk Keppens; Guglielmo Lunardon; Antonio Pasini; Stanley Payne; Ernie Shult; Koen Thas. 


\section{A final word (on Ernie, and others)}

During the editing process, Ernie Shult became very ill, to such an extent that he was not able to manage his own contribution to this volume in the way he wanted. He passed the files on to Koen, and Koen handled them from that moment on. In July 2015, Ernie passed away, surrounded by his children, and his wife Jiang. Ernie was one of Jef's frequent collaborators, and also one of his great friends; so we felt that at least some words for Ernie were in place here. One of Ernie's other famous "old" collaborators, Francis Buekenhout, also has a long paper in this volume, co-authored with Thomas Connor. It might be interesting to mention that Ernie published a paper with Koen, following a stay in Oberwolfach together with Stefaan De Winter, long after the Thas family visited Manhattan, Kansas in Summer 1991, when Koen was still a child.

Other families that were important in Jef Thas's mathematical and personal life were the Bruens, the Paynes and the Hirschfelds. Many people will have heard the true story of the Bruen and Thas families almost dying during a storm, while sailing in a little boat on a Canadian lake. There were also intense and long-lasting research collaborations with the Italian school of Finite Geometry, led by Guglielmo Lunardon, and with the Brussels geometry group of Francis Buekenhout.

All of these families and schools have members who lectured at the conference, a wonderful feat after all these years.

James, Koen and Hendrik 